\title{
An Attempt to Make Non-Elementary Functions That Are Giving Solutions to Some Second-Order Nonlinear Autonomous ODEs
}

\author{
Magne Stensland \\ Independent Researcher, Moldjord, Norway \\ Email: mag-ste@online.no
}

How to cite this paper: Stensland, M. (2022) An Attempt to Make Non-Elementary Functions That Are Giving Solutions to Some Second-Order Nonlinear Autonomous ODEs. Journal of Applied Mathematics and Physics, 10, 56-67.

https://doi.org/10.4236/jamp.2022.101006

Received: November 25, 2021

Accepted: January 15, 2022

Published: January 18, 2022

Copyright (c) 2022 by author(s) and Scientific Research Publishing Inc. This work is licensed under the Creative Commons Attribution International License (CC BY 4.0).

http://creativecommons.org/licenses/by/4.0/

\begin{abstract}
In this paper, we define an exponential function whose exponent is the product of a real number and the upper limit of integration in a non-elementary integral that can be arbitrary. We are using Abel's methods, described by Armitage and Eberlein. The key is to start with a non-elementary integral function, differentiating and inverting, and then define a set of functions. Differentiating these functions twice give second-order nonlinear ODEs that have the defined set of functions as solutions.
\end{abstract}

\section{Keywords}

Non-Elementary Functions, Second-Order Nonlinear Autonomous ODE

\section{Introduction}

On page 1 in the book [1], we find the sentences: Very few ordinary differential equations have explicit solutions expressible in finite terms. This is not because ingenuity fails, but because the repertory of standard functions (polynomials, exp, sin and so on) in terms of which solutions may be expressed is too limited to accommodate the variety of differential equations encountered in practice.

This is the main reason for this work. It should be possible to do something with this problem. If we don't have enough tools in our mathematical toolbox, we must make the tools first. For this problem, we will attempt to define some new functions. During a few years, I have succeeded to make more than 100 sets of non-elementary functions that are giving solutions to some hundred second-order nonlinear autonomous ODEs. In this paper, I want to share some of these results with you. Now, we will attempt to make some new functions. The numbers I have given the ODEs and the integral functions (IF) in the text are the number 
they have in my collection.

Wolfram Math World describes three nonlinear second-order ODEs that have the Jacobi elliptic functions $s n, c n$ and $d n$ as solutions. Define a solution $x(t)=c n(t)$ and differentiate twice, and you will obtain the ODE:

$$
\frac{\mathrm{d}^{2} x}{\mathrm{~d} t^{2}}=\left(2 k^{2}-1\right) x-2 k^{2} x^{3}, 0 \leq k<1
$$

And if we use the Jacobi amplitude function $a m(t, k)$ as a solution $x(t)$ and differentiate twice, we will obtain the ODE:

$$
\frac{\mathrm{d}^{2} x}{\mathrm{~d} t^{2}}=-k^{2} \sin (x) \cos (x)
$$

This causes us to think that other second-order nonlinear ODEs have functions made by the same methods than Jacobi elliptic functions, as their solutions. It should be possible to make more non-elementary functions by changing the non-elementary integral. In this paper, we will work in the same way: First, define some non-elementary functions, and then differentiating them twice in order to see what kind of ODEs these functions are giving solutions to.

We will use the methods described by Armitage and Eberlein [2] in their book Elliptic Functions, especially Section 1.6 and 1.7. They apply what they call the Abel's methods. "Eberlein sought to relate the ideas of Abel to the later work of Jacobi." Here is a brief summary of how they define the Jacobi elliptic functions:

In Section 1.6, they define a function $x$.

1) $x=x(\psi)=\int_{0}^{\psi} \frac{\mathrm{d} u}{\sqrt{1-k^{2} \sin ^{2} u}}, 0 \leq k<1,-\infty<\psi<\infty$

Here is $x$ a function of $\psi$. The positive derivative of $x$ is:

2) $\frac{\mathrm{d} x}{\mathrm{~d} \psi}=\frac{1}{\sqrt{1-k^{2} \sin ^{2} \psi}}$ and then inverting $\frac{\mathrm{d} x}{\mathrm{~d} \psi}$, so that

3) $\frac{\mathrm{d} \psi}{\mathrm{d} x}=\sqrt{1-k^{2} \sin ^{2} \psi}$ here is $\psi$ a function of $x$.

And then in Section 1.7, they define a set of three functions $s n, c n$ and $d n$, so that

$$
\operatorname{sn}(x)=\sin \psi, \operatorname{cn}(x)=\cos \psi \text { and } d n(x)=\sqrt{1-k^{2} \sin ^{2} \psi}
$$

Armitage and Eberlein are using $x$ as variable to the functions $s n, c n$ and $d n$. In order to avoid misunderstandings with the solution $x(t)$, we will use the variable $u$ instead $x$, and the amplitude $\phi$ instead of $\psi$.

During the last 30 years, there have been done a lot of progress in finding solutions to nonlinear ODEs and PDEs. The progress is mostly made by using different methods like the Prelle-Singer method [3], Abel's equations [4] [5], the new Jacobi elliptic functions [6] [7], the old Jacobi elliptic functions [8] [9], the Prelle-Singer method [10] [11], a new method [12], revised methods [13].

With exception from the new Jacobi elliptic functions, it seems to me that nobody has tried to make new non-elementary functions that can give solutions 
to second-order nonlinear ODEs. In this paper, we will attempt to take a step further.

The functions defined in this paper are new to the literature, at least to my knowledge.

\section{The Expo-Elliptic Functions}

This is a large group of functions and very useful as solutions to both secondorder nonlinear ODEs, and systems of ODEs. In this paper we will study two examples.

\subsection{Definition}

Define an exponential function $\mu=\mu(u)=\mathrm{e}^{a \varphi}, a, \varphi \in R,-\infty<a, \varphi<\infty$, $\varphi=\varphi(u)$ is the amplitude or upper limit of integration in a non-elementary integral. This connection to the elliptic functions is the reason for this special name. They may also be named $\mu$-functions, but that name tells nothing about these functions. The integral may also be elementary. Then the solution $x(t)=\mathrm{e}^{a \varphi}$ is an elementary solution. When $\frac{\mathrm{d} \varphi}{\mathrm{d} t}=1$, is $\mathrm{e}^{a \varphi}=\mathrm{e}^{a t}$. The expo-elliptic function is more general than the elementary exponential function. The expo-elliptic function is not periodic, and it is continuous and differentiable on the whole $R$, for the limitations of the parameters.

\subsection{The Derivative of the Expo-Elliptic Function}

$$
\frac{\mathrm{d}}{\mathrm{d} u} \mu(u)=a \mathrm{e}^{a \varphi} \frac{\mathrm{d} \varphi}{\mathrm{d} u}=a \mu(u) \frac{\mathrm{d} \varphi}{\mathrm{d} u}
$$

\subsection{Two Examples}

Let us take a look at two examples of expo-elliptic functions, and some second-order ODEs that have these functions as solutions. I think that the behavior of the solution curves in a phase diagram reflect the qualities of the solution functions. And by considering the behavior of the solution curves we can discover some of the qualities of the expo-elliptic functions.

\subsubsection{The Functions $p s$ and $p d$}

Just like the Jacobi elliptic functions we start with a non-elementary integral.

Define an integral function $u$ (IF 118):

$$
u=u(\varphi)=\int_{0}^{\varphi} \frac{\mathrm{e}^{a \theta}}{n+h \cos \left(p \mathrm{e}^{a \theta}+b\right)+k \sin \left(v \mathrm{e}^{a \theta}+d\right)} \mathrm{d} \theta
$$

$a, b, d, h, k, n, p, v$ are parameters defined on $R$.

The denominator can become 0 . In order to avoid that we can make some restrictions to the parameters: $n>2,-1 \leq h, k \leq 1$

If we use the substitution $y=\mathrm{e}^{a \theta}, \mathrm{d} \theta=\frac{\mathrm{d} y}{a y}$, 
we become the integral: $\frac{1}{a} \int_{0}^{\varphi} \frac{\mathrm{d} y}{n+h \cos (p y+b)+k \sin (v y+d)}$

According to integral-calculator.com this is a non-elementary integral.

$$
\frac{\mathrm{d} u}{\mathrm{~d} \varphi}=\frac{\mathrm{e}^{a \varphi}}{n+h \cos \left(p \mathrm{e}^{a \varphi}+b\right)+k \sin \left(v \mathrm{e}^{a \varphi}+d\right)}
$$

Inverting $\frac{\mathrm{d} u}{\mathrm{~d} \varphi}$ :

$$
\frac{\mathrm{d} \varphi}{\mathrm{d} u}=\mathrm{e}^{-a \varphi}\left(n+h \cos \left(p \mathrm{e}^{a \varphi}+b\right)+k \sin \left(v \mathrm{e}^{a \varphi}+d\right)\right)
$$

Then we define a set of 2 functions $p s$ and $p d$, so that

$$
\operatorname{ps}(u)=\mathrm{e}^{a \varphi}
$$

And

$$
\begin{aligned}
p d(u) & =\mathrm{e}^{-a \varphi}\left(n+h \cos \left(p \mathrm{e}^{a \varphi}+b\right)+k \sin \left(v \mathrm{e}^{a \varphi}+d\right)\right) \\
& =\frac{1}{p s(u)}(n+h \cos (p p s(u)+b)+k \sin (v p s(u)+d))
\end{aligned}
$$

The connection between the functions $p s$ and $p d$ is:

$$
p d(u) p s(u)-h \cos (p p s(u)+b)-k \sin (v p s(u)+d)=n
$$

We see that the function $p d(u)$ exist for all values of the parameters $a, b, d, h$, $k, n, p, v$, even though the integral IF 118 don't exist for values that make the denominator $=0$. The functions $p s(u)$ and $p d(u)$ are continues and differentiable on the whole $R$.

The derivatives to these functions are:

$$
\begin{aligned}
& \frac{\mathrm{d}}{\mathrm{d} u} p s(u)=a \mathrm{e}^{a \varphi} \frac{\mathrm{d} \varphi}{\mathrm{d} u}=\operatorname{aps}(u) p d(u) \\
\frac{\mathrm{d}}{\mathrm{d} u} p d(u)= & -\frac{a}{p s^{2}(u)}(n+h \cos (p p s(u)+b)+k \sin (v p s(u)+d))^{2} \\
+ & \frac{a}{p s(u)}(n+h \cos (p p s(u)+b)+k \sin (v p s(u)+d)) \\
& \times[k v \cos (v p s(u)+d)-h p \sin (p p s(u)+b)]
\end{aligned}
$$

We can find out which second-order nonlinear ODEs the $p s$-function give solution to, by differentiating the function $p s(t)$ twice.

Define a solution $x(t)=p s(t)$

$$
\begin{gathered}
\frac{\mathrm{d} x}{\mathrm{~d} t}=a p s(t) p d(t)=a x p d(t)=a(n+h \cos (p x+b)+k \sin (v x+d)) \\
\frac{\mathrm{d}^{2} x}{\mathrm{~d} t^{2}}=-a h p \frac{\mathrm{d} x}{\mathrm{~d} t} \sin (p x+b)+a k v \frac{\mathrm{d} x}{\mathrm{~d} t} \cos (v x+d)
\end{gathered}
$$

(2779) $\frac{\mathrm{d}^{2} x}{\mathrm{~d} t^{2}}=a^{2}(n+h \cos (p x+b)+k \sin (v x+d))[k v \cos (v x+d)-h p \sin (p x+b)]$ 
(2780) $\frac{\mathrm{d}^{2} x}{\mathrm{~d} t^{2}}=a \frac{\mathrm{d} x}{\mathrm{~d} t} k v \cos (v x+d)-a^{2} h p \sin (p x+b)(n+h \cos (p x+b)+k \sin (v x+d))$

Figure 1 and Figure 2 show phase diagrams of Equation (2780) with different values of the parameters.

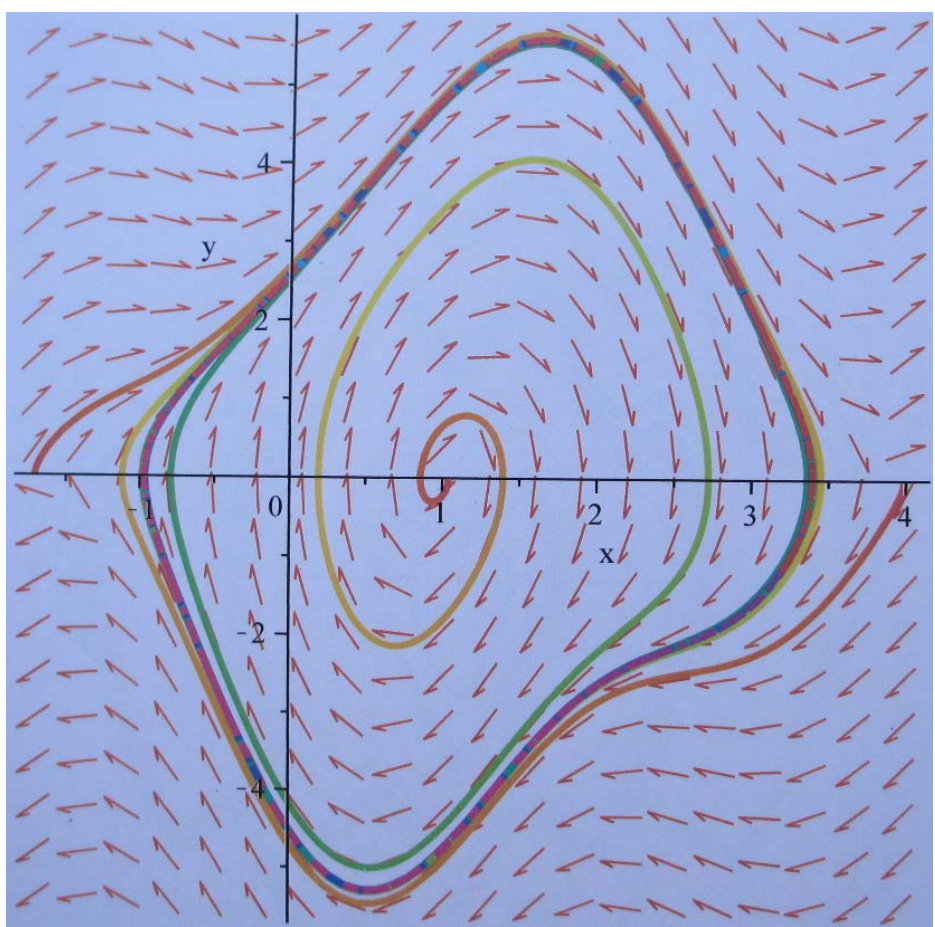

Figure 1. One LC.

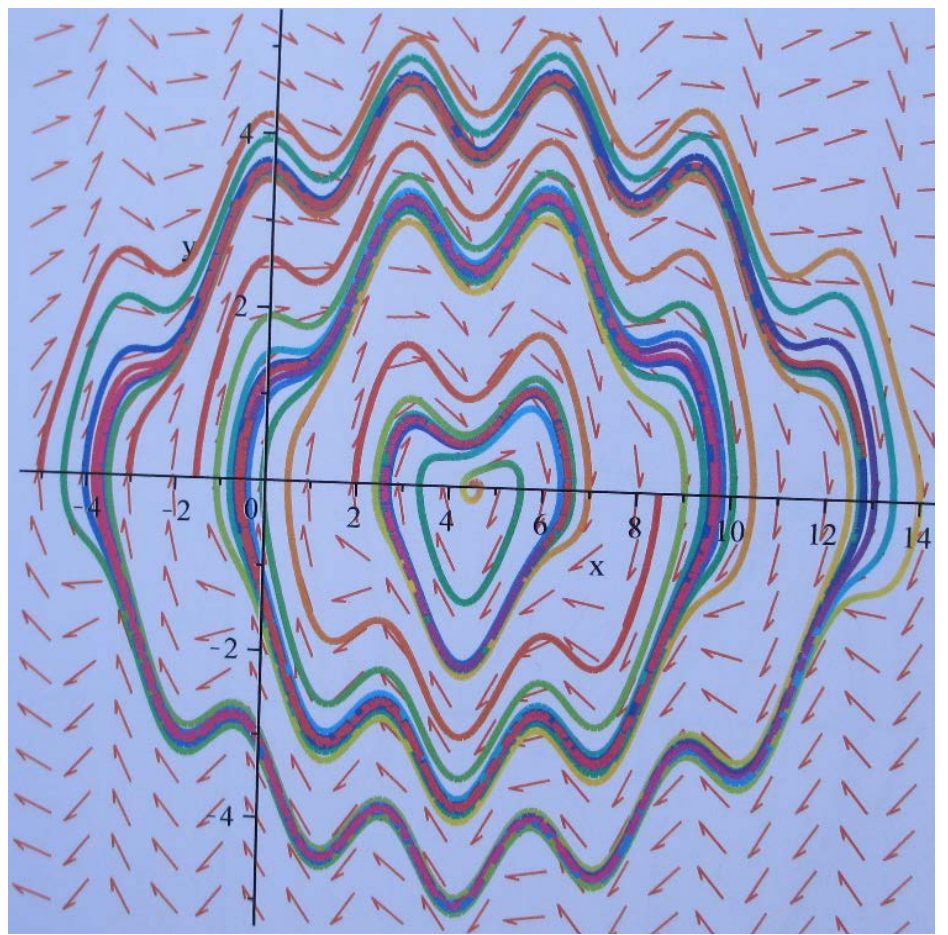

Figure 2. Three LC. 
In Figure 1, the parameters have the values $a=1, k=1, v=2, b=-1, p=1, d$ $=1, v=2, n=3, h=2$.

A limit cycle (LC) that reminds a bit of van der Pol's LC.

In Figure 2, the parameters have the values $a=-1, k=1 / 2, v=2, p=1 / 4, n=$ $6, b=2, d=-1, h=-1$.

Three stable limit cycles inside each other with the same equilibrium point. Between the stable limit cycles there are unstable limit cycles [1].

If we use $\frac{\mathrm{d} x}{\mathrm{~d} t}=\operatorname{aps}(t) p d(t)$ and differentiating one more time, we get the equation

$$
\begin{aligned}
\frac{\mathrm{d}^{2} x}{\mathrm{~d} t^{2}}= & \frac{1}{x}\left(\frac{\mathrm{d} x}{\mathrm{~d} t}\right)^{2}-\frac{a}{x} \frac{\mathrm{d} x}{\mathrm{~d} t}(n+h \cos (p x+b)+k \sin (v x+d))+a k v \frac{\mathrm{d} x}{\mathrm{~d} t} \cos (v x+d) \\
& -a^{2} h p \sin (p x+b)(n+h \cos (p x+b)+k \sin (v x+d))
\end{aligned}
$$

In Equation (2784) $\frac{\mathrm{d}^{2} x}{\mathrm{~d} t^{2}}$ don't exist when $x=0$, except in one point $(0, a(n+h \cos (b)+k \sin (d)))$. In Figure 3, we see that the solution curves are crossing the asymptote $x=0$ in one point, and they also intersect in the same point. This is an example of when the Uniqueness Theorem fails. The derivatives don't exist everywhere in the neighbourhood of $(0, a(n+h \cos (b)+k \sin (d)))$. This special phenomenon I have named "hole in asymptote".

In Figure 3, the parameters have the values $a=1, n=3, h=k=p=1, v=2, d$ $=-1, b=-1 / 4$.

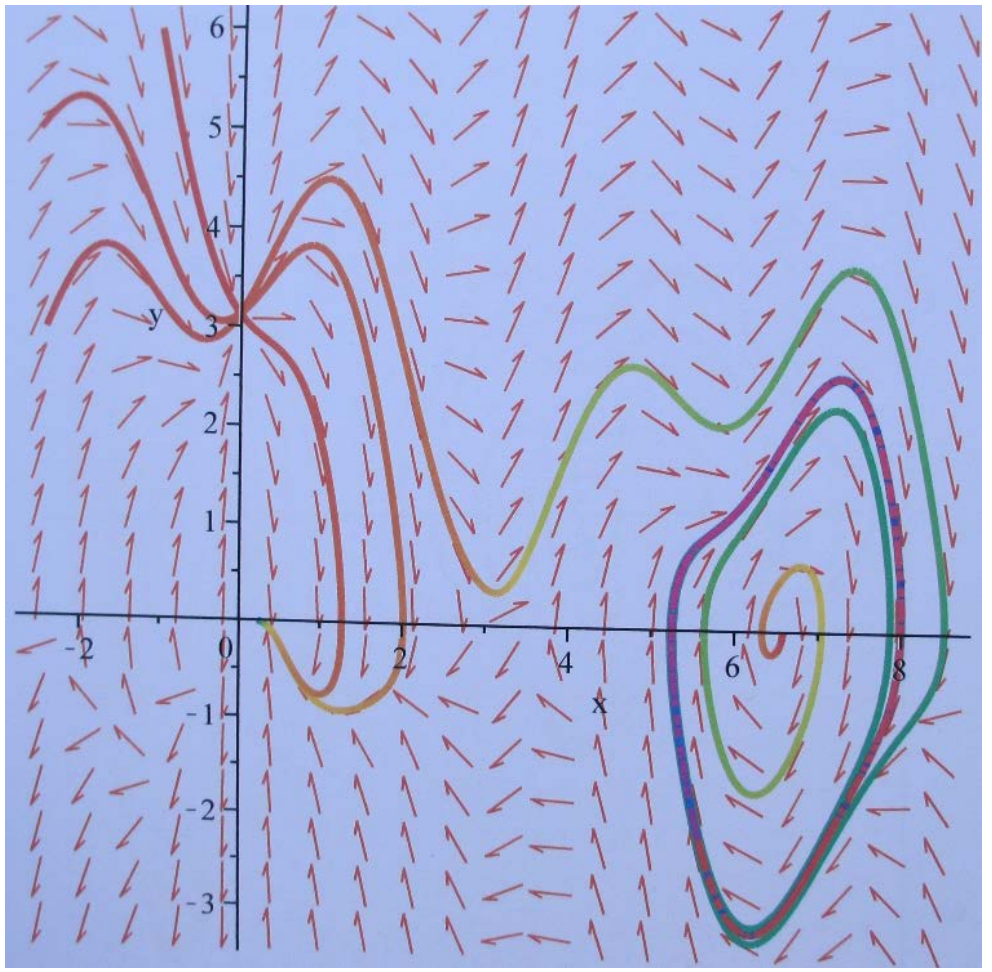

Figure 3. Hole in asymptote. 
The $p s$-function is giving solutions to a lot of other second-order nonlinear ODEs and systems of ODEs. In the three pictures above we can see some of the qualities of the functions $p s$ and $p d$.

\subsubsection{The Functions $h s$ and $h d$}

This second example of expo-elliptic functions also starts with a non-elementary integral.

Define a function $u$ (IF 180):

$$
u=u(\varphi)=\int_{0}^{\varphi} \frac{\mathrm{e}^{a \theta}}{n+h \cos \left(s \mathrm{e}^{2 a \theta}+b\right)+f \sin \left(q \mathrm{e}^{2 a \theta}+g\right)+k \mathrm{e}^{a \theta} \sin \left(p \mathrm{e}^{a \theta}+c\right) \cos \left(v e^{a \theta}+d\right)} \mathrm{d} \theta
$$

The denominator can become 0 . To avoid that, we can make some restrictions to the parameters: $a<0, n>3,-1 \leq f, h, k \leq 1$, $a, b, c, d, g, h, k, n, s, p, v, q \in R$

$$
\frac{\mathrm{d} u}{\mathrm{~d} \varphi}=\frac{\mathrm{e}^{a \varphi}}{n+h \cos \left(\mathrm{se}^{2 a \varphi}+b\right)+f \sin \left(q \mathrm{e}^{2 a \varphi}+g\right)+k \mathrm{e}^{a \varphi} \sin \left(p \mathrm{e}^{a \varphi}+c\right) \cos \left(v \mathrm{e}^{a \varphi}+d\right)}
$$

Inverting:

$$
\begin{aligned}
\frac{\mathrm{d} \varphi}{\mathrm{d} u}= & \mathrm{e}^{-a \varphi}\left(n+h \cos \left(\mathrm{se}^{2 a \varphi}+b\right)+f \sin \left(q \mathrm{e}^{2 a \varphi}+g\right)\right. \\
& \left.+k \mathrm{e}^{a \varphi} \sin \left(p \mathrm{e}^{a \varphi}+c\right) \cos \left(v \mathrm{e}^{a \varphi}+d\right)\right)
\end{aligned}
$$

Define a set of 2 functions: $h s$ and $h d$, so that

$$
\begin{gathered}
h s(u)=\mathrm{e}^{a \varphi}, \\
h d(u)=\frac{\mathrm{d} \varphi}{\mathrm{d} u}=\frac{1}{h s(u)}\left(n+h \cos \left(s h s^{2}(u)+b\right)+f \sin \left(q h s^{2}(u)+g\right)\right. \\
+k h s(u) \sin (p h s(u)+c) \cos (v h s(u)+d))
\end{gathered}
$$

The connection between these functions is:

$$
\begin{aligned}
& h d(u) h s(u)-h \cos \left(s h s^{2}(u)+b\right)-f \sin \left(q h s^{2}(u)+g\right) \\
& -k h s(u) \sin (p h s(u)+c) \cos (v h s(u)+d)=n
\end{aligned}
$$

Notice that the functions $h s$ and $h d$ exist for all values of the parameters $a, b, c$, $d, g, h, k, n, s, p, v, q$ even though the integral IF 180 is not defined for values that makes the denominator $=0$. The functions $h s$ and $h d$ are continuous and differentiable on the whole $R$.

The derivatives to these functions are:

$$
\begin{gathered}
\frac{\mathrm{d}}{\mathrm{d} u} h s(u)=a \mathrm{e}^{a \varphi} \frac{\mathrm{d} \varphi}{\mathrm{d} u}=a h s(u) h d(u) \\
\frac{\mathrm{d}}{\mathrm{d} u} h d(u)=-\frac{a}{h s^{2}(u)}\left(n+h \cos \left(s h s^{2}(u)+b\right)+f \sin \left(q h s^{2}(u)+g\right)\right. \\
+k h s(u) \sin (p h s(u)+c) \cos (v h s(u)+d))^{2} \\
+\frac{a}{h s(u)}\left(n+h \cos \left(\operatorname{shs}^{2}(u)+b\right)+f \sin \left(q h s^{2}(u)+g\right)\right.
\end{gathered}
$$




$$
\begin{aligned}
& +k h s(u) \sin (p h s(u)+c) \cos (v h s(u)+d)) \\
& \times\left[-2 h s h s(u) \sin \left(s h s^{2}(u)+b\right)+2 f q h s(u) \cos \left(q h s^{2}(u)+g\right)\right. \\
& +k \sin (p h s(u)+c) \cos (v h s(u)+d)+k p h s(u) \cos (p h s(u)+c) \\
& \times \cos (v h s(u)+d)-k v h s(u) \sin (p h s(u)+c) \sin (v h s(u)+d)]
\end{aligned}
$$

Define a solution $x(t)=h s(t)$

$$
\begin{aligned}
\frac{\mathrm{d} x}{\mathrm{~d} t}=a( & \left(n+h \cos \left(s x^{2}+b\right)+f \sin \left(q x^{2}+g\right)+k x \sin (p x+c) \cos (v x+d)\right) \\
\frac{\mathrm{d}^{2} x}{\mathrm{~d} t^{2}}= & 2 a x \frac{\mathrm{d} x}{\mathrm{~d} t}\left(f q \cos \left(q x^{2}+g\right)-h s \sin \left(s x^{2}+b\right)\right)+a^{2} k\left(n+h \cos \left(s x^{2}+b\right)\right. \\
& \left.+f \sin \left(q x^{2}+g\right)+k x \sin (p x+c) \cos (v x+c)\right)[\sin (p x+c) \cos (v x+d) \\
& +p x \cos (p x+c) \cos (v x+d)-v x \sin (p x+c) \sin (v x+d)]
\end{aligned}
$$

$(0,0)$ is equilibrium point when $c=0$. We will use this in the equations below. Define 2 functions $f$ and $g$, so that $f(x, y)=y$ and

$$
\begin{aligned}
g(x, y)= & 2 a x y\left(f q \cos \left(q x^{2}+g\right)-h \sin \left(s x^{2}+b\right)\right)+a^{2} k\left(n+h \cos \left(s x^{2}+b\right)\right. \\
& \left.+f \sin \left(q x^{2}+g\right)+k x \sin (p x) \cos (v x+d)\right)[\sin (p x) \cos (v x+d) \\
& +p x \cos (p x) \cos (v x+d)-v x \sin (p x) \sin (v x+d)]
\end{aligned}
$$

Jacobian matrix:

$$
\begin{gathered}
J=\left[\begin{array}{ll}
\frac{\partial f}{\partial x}\left(x_{0}, y_{0}\right) & \frac{\partial f}{\partial y}\left(x_{0}, y_{0}\right) \\
\frac{\partial g}{\partial x}\left(x_{0}, y_{0}\right) & \frac{\partial g}{\partial y}\left(x_{0}, y_{0}\right)
\end{array}\right] \\
\operatorname{det}(J(0,0)-\lambda I)=\operatorname{det}\left[\begin{array}{cc}
0-\lambda \\
2 a^{2} k p \cos (d)(n+h \cos (b)+f \sin (g)) & 0-\lambda
\end{array}\right] \\
(-\lambda)^{2}-2 a^{2} k p \cos (d)(n+h \cos (b)+f \sin (g))=0 \\
\lambda= \pm a \sqrt{2 k p \cos (d)(n+h \cos (b)+f \sin (g))}
\end{gathered}
$$

Complex eigenvalues with zero real part when

$k p \cos (d)(n+h \cos (b)+f \sin (g))<0$

That means $(0,0)$ is center.

Let us take a look at some phase diagrams of Equation (3341):

In Figure 4 is $(0,0)$ an "unstable center", with an outwards rotating spiral, ending up on a limit cycle (LC) to the right. I have named this special equilibrium point "unstable center", because the eigenvalues of the Jacobian matrix at $(0,0)$ are complex with zero real part, even though the pictures show spirals around $(0,0)$. An example of when linearization fails.

In Figure 5, we have changed the value of the parameter a from +1 in Figure 4 to -1 in Figure 5. $(0,0)$ is still an unstable center, but now with an inwards rotating spiral, ending up in $(0,0)$ in infinity. A large LC is surrounding the un- 
stable center and 2 spirals. This is an example of bifurcation with the parameter a. In Figure 5, we have these equilibrium points: One unstable center (spiral sink), one spiral source with a stable LC around to the left, one spiral sink to the right surrounded by an unstable LC and a stable LC outside it again, 2 saddle points and 3 limit cycles.

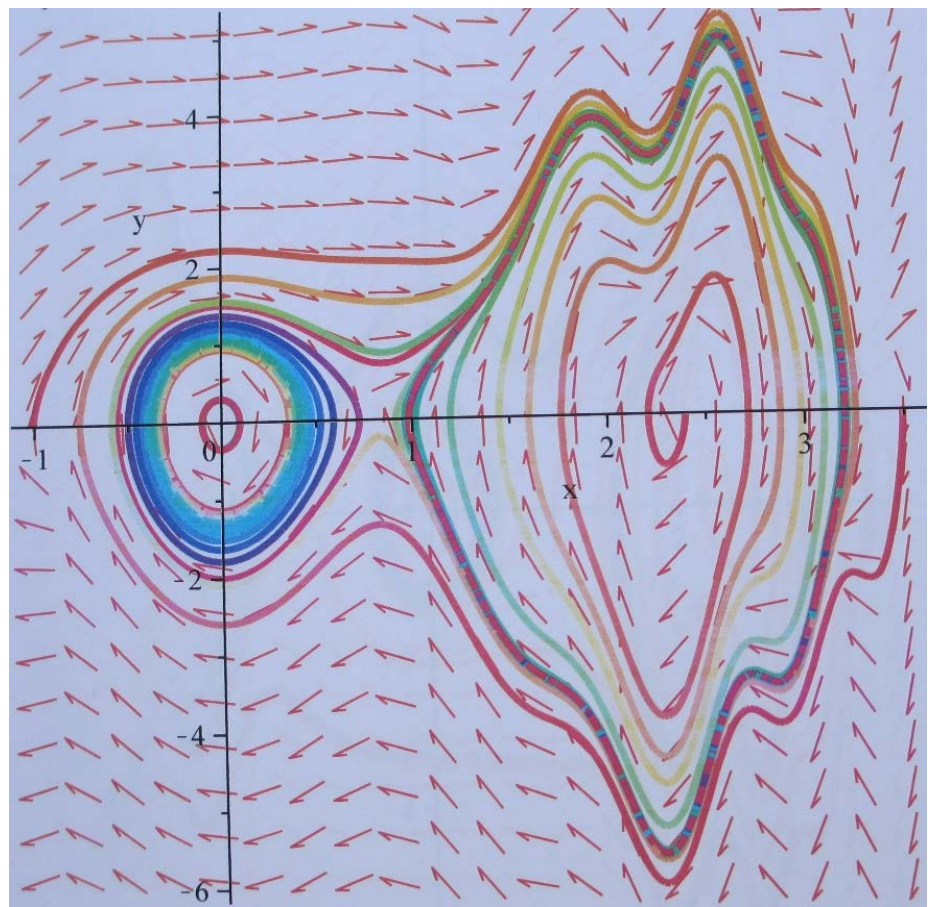

Figure 4. One unstable center and one LC.

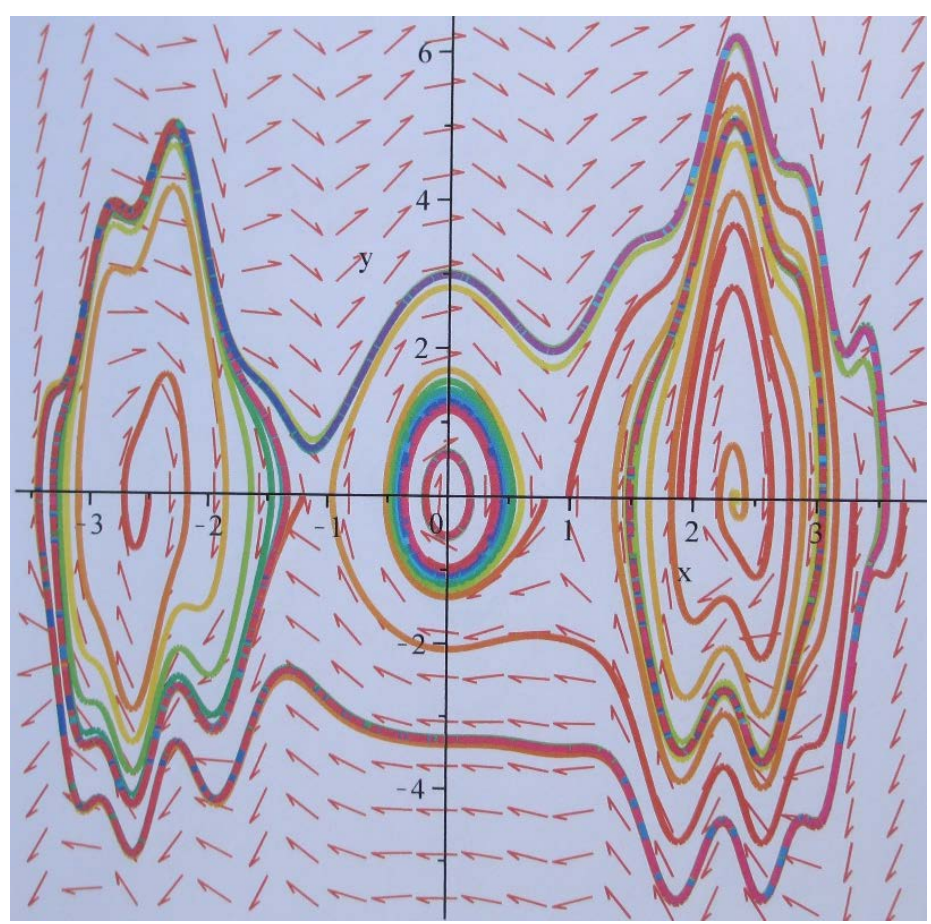

Figure 5. One unstable center and three LC. 
In Figure 6 is the parameter $d=0 .(0,0)$ is now a stable center, with stable closed curves around. And we see stable closed curves with no spiraling behavior, where we in Figure 5 saw a large LC. In Figure 6, we find these equilibrium points: One stable center, one spiral sink to the left, one spiral source to the right surrounded by a LC, and two saddle points. All these are surrounded by stable closed curves.

Consider Figure 7 below and compare with Figure 4. The only difference is the parameter $d$, which is zero in Figure 7. Bifurcation with the parameter $d$.

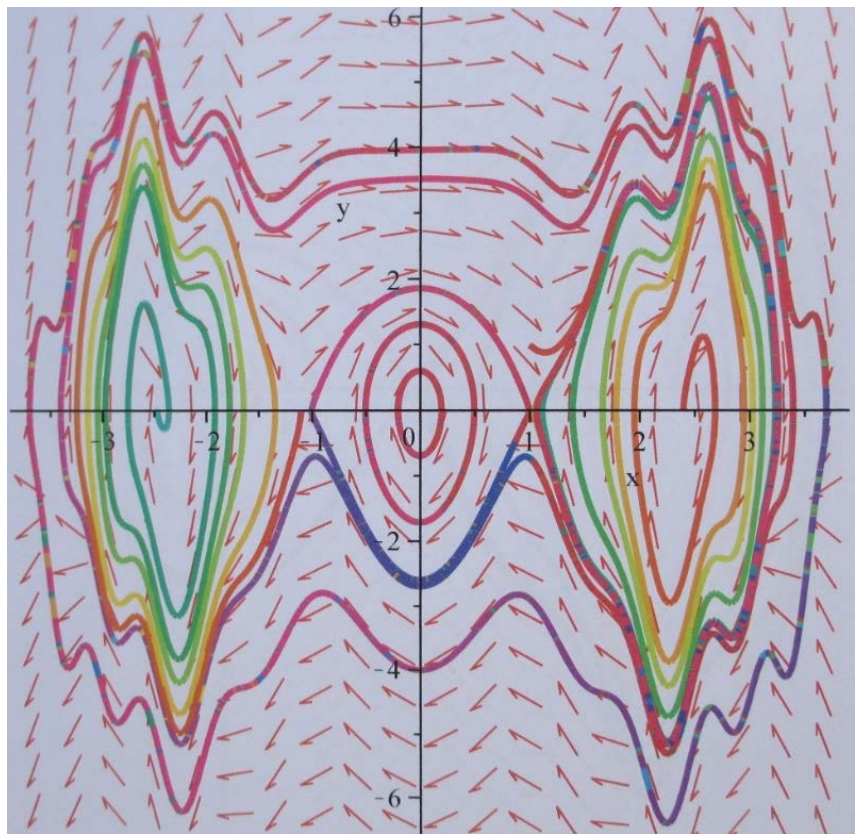

Figure 6. One stable center with stable closed curves.

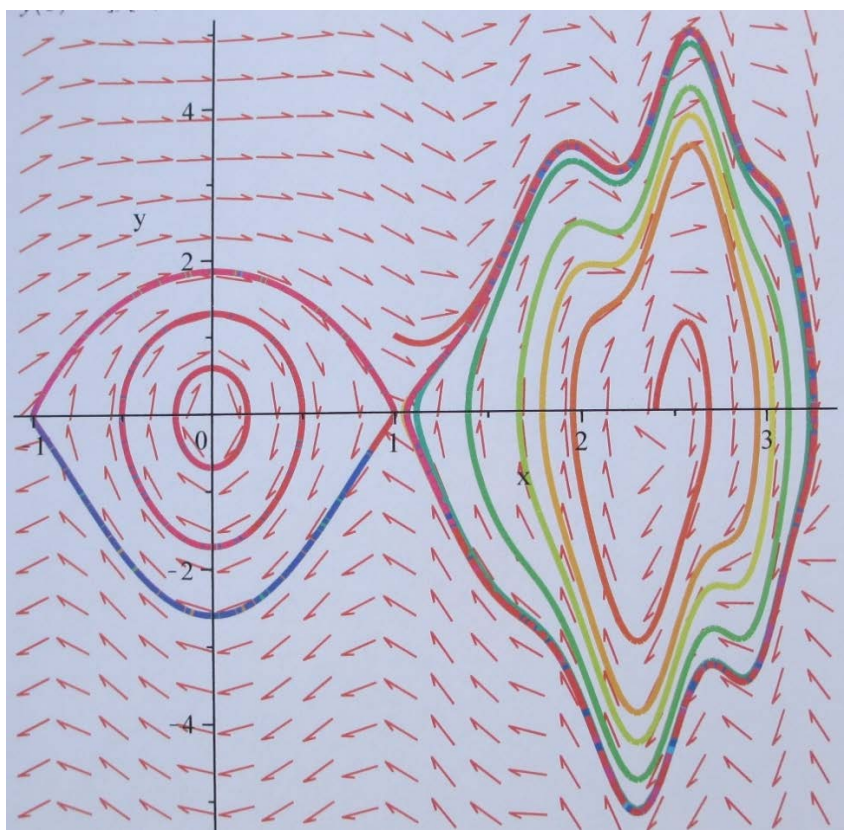

Figure 7. One stable center and one LC. 
It is possible to make many other non-elementary functions from the functions $h s$ and $h d$ by choosing one or more of the parameters $=0$. These special cases are a bit easier to work with, but they can have a lot of funny and interesting behavior.

\section{Conclusions}

My purpose with all these pictures is to show you some of the variety of behavior and qualities of the expo-elliptic functions. I don't know any other functions that have these qualities. If the behavior of the solution curves in the phase diagrams reflects the qualities of the solution functions, then we can see some of these qualities in the pictures in this paper.

It is possible to make a lot of non-elementary functions using the Abel's methods described by Armitage and Eberlein, by how they define the Jacobi elliptic functions. In the same way as Jacobi's functions $s n, c n, d n$ and am give solutions to a few ODEs, the expo-elliptic functions described in this paper and a lot more, give solutions to many other different kind of ODEs. I don't see any limit for this subject. The only limit is our imagination.

\section{Conflicts of Interest}

The author declares no conflicts of interest regarding the publication of this paper.

\section{References}

[1] Jordan, D.W. and Smith, P. (2007) Nonlinear Ordinary Differential Equations. Oxford University Press, Oxford, 1, 450-453, 493.

[2] Armitage, J.V. and Eberlein, W.F. (2006) Elliptic Functions. London Mathematical Society, Cambridge, 12-15.

[3] Duarte, L.G.S., Duarte, S.E.S., da Mota, L.A.C.P. and Skea, J.E.F. (2000) Solving Second-Order Ordinary Differential Equations by Extending the Prelle-Singer Method. Journal of Physics A: Mathematical and General, 34, 3015. https://doi.org/10.1088/0305-4470/34/14/308

[4] Markakis, M.P. (2010) Exact Solutions for Certain Nonlinear Autonomous ODE of the Second Order and Families of Two-Dimensional Autonomous Systems. International Journal of Differential Equations, 2010, Article ID: 436860. https://doi.org/10.1155/2010/436860

[5] Panayotounakos, D.E. and Zampoutis, T.I. (2011) Construction of Exact Parametric or Closed Form Solutions of Some Unsolvable Classes of Nonlinear ODEs. International Journal of Mathematics and Mathematical Sciences, 2011, Article ID: 387429. https://doi.org/10.1155/2011/387429

[6] Ali, A.T. (2011) New Generalized Jacobi Elliptic Function Rational Expansion Method. Journal of Computation and Applied Mathematics, 235, 4117-4127.

[7] Hong, B.J. and Lu, D.C. (2009) New Jacobi Elliptic Solutions for Higher-Order Nonlinear Schrodinger Equation. International Journal of Nonlinear Science, 7, 360-367.

[8] Schwalm, W.A. (2015) Differential Equations Satisfied by the Jacobi Elliptic Functions. Morgan \& Claypool Publishers, San Rafael. 
https://doi.org/10.1088/978-1-6817-4230-4ch2

[9] Salas, A.H. and Castillo H., J.E. (2014) Exact Solution to Duffing Equation and the Pendulum Equation. Applied Mathematical Sciences, 8, 8781-8789.

https://doi.org/10.12988/ams.2014.44243

[10] Chandrasakar, V.K., Senthilvelan, M. and Lakshmanan, M. (2005) On the Complete Integrability and Linearization of Certain Second-Order Nonlinear Ordinary Differential Equations. Proceedings of the Royal Society $A, 461$.

https://doi.org/10.1098/rspa.2005.1465

[11] Chandrasakar, V.K., Senthilvelan, M. and Lakshmanan, M. (2008) On the Complete Integrability and Linearization of Nonlinear Ordinary Differential Equations. Proceedings of the RoyaL Society A, 461.

https://doi.org/10.1098/rspa.2008.0240

[12] Zraiqat, A. and Al-Hwawcha, L.K. (2015) On Exact Solutions of Second Order Nonlinear Ordinary Differential Equations. Applied Mathematics, 6, 953-957. https://doi.org/10.4236/am.2015.66087

[13] Mengesha, L.M. and Denekew, S.A. (2020) Revised Methods for Solving Nonlinear Second Order Differential Equations. Journal of Applied \& Computational Mathematics, 9, 4 . 